УДК 512.64

O. G. Nakonechniy, G. I. Kudin, T. P. Zinko

\title{
FORMULAS OF PERTURBATION FOR ONE CLASS OF PSEUDO INVERSE OPERATORS
}

\begin{abstract}
O. G. Nakonechniy, G. I. Kudin, T. P. Zinko. Formulas of perturbation for one class of pseudo inverse operators, Mat. Stud. 52 (2019), 124-132.

Pseudo inverse matrix research, as well as related issue of their application, is in focus of scientific literature recently. Moreover, the field of applications of pseudo inverse matrices is expanding and obtained theoretical results are successfully used to solve practical problems. Interest in the pseudo inverse matric problem is large due to their various applications: in control theory, identification problems, approximation theory, statistical problems, theory of recurrent filtration and others.

We investigate a linear operator equation, where the operator is a linear combination of matrices. We obtain solution using the pseudo inverse operation and small parameter method.
\end{abstract}

Research subject of this publication is the application of the pseudo inverse matrix technique for solving linear regression analysis problems under the conditions when matrices are observing elements. Such problems appear in recognition and classification tasks with matrix features. The first variant of an application of pseudo-inversion matrix in matrix Euclidean spaces is Klein formula [1, 2]. We note that among the publications of recent years there is a work [7] devoted to an application of pseudo inverse technique in matrix Euclidean spaces.

1. Formulation of the problem of perturbation of a linear operator from vector space $\mathbb{R}^{n}$ to the space of matrices $\mathbb{R}^{m \times n}$. Let $A_{k}, k \in \overline{1, s}$ be a sequence of matrices of dimension $(m \times n)$, elements of matrix space $\mathbb{R}^{m \times n}$, sequence of numbers $y_{k}, k \in \overline{1, s}$. We consider the operator equation

$$
\wp y \equiv \sum_{k=1}^{K} A_{k} y_{k}=Y,
$$

where $y=\left(y_{1}, \ldots, y_{K}\right)^{T} \in \mathbb{R}^{K}$ is an unknown vector, given matrix $Y \in \mathbb{R}^{m \times n}$, linear operator $\wp y$, which acts from a finite dimensional Euclidean vector space $\mathbb{R}^{s}$ into another Euclidean space of matrices $\mathbb{R}^{m \times n}$.

We investigate the problems of the vector decomposition in this paper

$$
y(\varepsilon)=\left(y_{1}(\varepsilon), \ldots, y_{K}(\varepsilon)\right)^{T} \in \mathbb{R}^{K},
$$

which is a solution of the operator equation

$$
\left(\wp+\varepsilon \wp_{1}\right) y(\varepsilon) \equiv \sum_{k=1}^{s}\left(A_{k}+\varepsilon A_{k}(1)\right) y_{k}(\varepsilon)=Y,
$$

2010 Mathematics Subject Classification: 15Bxx.

Keywords: vector decomposition; pseudo inverse; linear operator; SVD; perturbed operator. doi:10.30970/ms.52.2.124-132

(C) O. G. Nakonechniy, G. I. Kudin, T. P. Zinko, 2019 


$$
A_{k}, A_{k}(1) \in \mathbb{R}^{m \times n}, k \in \overline{1, s},
$$

for a small parameter $\varepsilon(0<\varepsilon<1)$.

According to the theory of pseudo-inversion in Euclidean matrix spaces [7], the solution of equation (1) can be written as

$$
y=\wp^{+} Y+Z(\wp) \mathbb{R}^{s}
$$

where $\wp^{+}$is the pseudo inverse operator of the operator $\wp, Z(\wp)$ is the orthogonal projector on operator's kernel of $\wp$.

A solution of the operator equation (2) in the first approximation of the small parameter method $y_{\varepsilon}=y+\varepsilon y(1)+o(\varepsilon)$ we obtain provided that at $\varepsilon=0$ the system of equations (2) coincides with the system of equations (1) for which we know the solution in the form (3).

1.1. Algorithm of the stated problem solution. According to the assumption that we know the solution of equation (1), and within the theory of pseudo-inversion, we can assume that we know SVD representation of the acting operator $\wp$, as well as pseudo inverse to it, projection operators.

Thus, in terms of the pseudo inverse theory in Euclidean space for the problem in question, we consider the operator $\wp: \mathbb{R}^{s} \rightarrow \mathbb{R}^{m \times n}$ and its adjoint $\wp^{*}: \mathbb{R}^{m \times n} \rightarrow \mathbb{R}^{s}$. Adjoint $\wp^{*}$ to the operator $\wp$ is a linear operator acting in the opposite direction to $\wp_{\alpha}$ [7]:

$$
\wp^{*} Y=\left(\begin{array}{c}
\left(A_{1}, Y\right)_{\operatorname{tr}} \\
\vdots \\
\left(A_{s}, Y\right)_{\operatorname{tr}}
\end{array}\right)=\left(\begin{array}{c}
\operatorname{tr}\left(Y^{T} A_{1}\right) \\
\vdots \\
\operatorname{tr}\left(Y^{T} A_{s}\right)
\end{array}\right), \quad Y \in \mathbb{R}^{m \times n} .
$$

In the formula (4) we use the scalar product in the matrix space $\mathbb{R}^{m \times n}$, a trace scalar product.

The product of two operators $\wp^{*} \wp: \mathbb{R}^{s} \rightarrow \mathbb{R}^{s}$ is a linear operator, generated by a matrix, defined by the relation:

$$
\wp^{*} \wp \equiv F=\left(\left(A_{i}, A_{j}\right)_{\operatorname{tr}}\right)_{i, j=1, s)} \equiv\left(\begin{array}{ccc}
\operatorname{tr} A_{1}^{T} A_{1} & \cdots & \operatorname{tr} A_{1}^{T} A_{s} \\
\vdots & \ddots & \vdots \\
\operatorname{tr} A_{s}^{T} A_{1} & \cdots & \operatorname{tr} A_{s}^{T} A_{s}
\end{array}\right) .
$$

For an arbitrary linear operator between two Euclidean spaces $\wp$ and its adjoint $\wp^{*}$ there are sets of singularities

$$
\left(\nu_{j}, \lambda_{j}^{2}\right),\left(U_{j}, \lambda_{j}^{2}\right), \lambda_{1} \geq \lambda_{2} \geq \ldots \geq \lambda_{r}>0, j \in \overline{1, r}, r=\operatorname{rang} F,
$$

of operators $\wp^{*} \wp$ and $\wp \wp^{*}$, respectively, with common set of eigenvalues for both operators, which completely determine the actions of operators $\wp^{*}, \wp$ themselves and with related operators. Since matrix (5) is a Gram matrix for the sequence of matrices $A_{k}, k \in \overline{1, s}$, then the value of the parameter $r=\operatorname{rank} F$ determines the number of linearly independent matrices of the sequence of matrices $A_{k}, k \in \overline{1, s}$.

The singular operator decomposition of $\wp^{*} \wp$ is determined by a set of eigenvalues and eigenvectors of the matrix $F \in \mathbb{R}^{s \times s}-\left(\nu_{j}, \lambda_{j}^{2}\right), j \in \overline{1, r}, r=\operatorname{rang} F$ :

$$
\nu_{q}^{T} \nu_{j}=\delta_{q j}, q \in \overline{1, r}, j \in \overline{1, r}, \lambda_{1} \geq \lambda_{2} \geq \ldots \geq \lambda_{r}>0,
$$




$$
\wp^{*} \wp \nu_{j} \equiv F \nu_{j}=\lambda_{j}^{2} \nu_{j}, j \in \overline{1, r} .
$$

The operator itself has the form

$$
\wp^{*} \wp \equiv F=\sum_{j=1}^{r} \lambda_{j}^{2} \nu_{j} \nu_{j}^{T}
$$

An element of a complete set of singularities $\left(U_{j}, \lambda_{j}^{2}\right), j \in \overline{1, r}$ of the operator $\wp \wp^{*}$ of matrix $U_{j} \in \mathbb{R}^{m \times n}, j \in \overline{1, r}$ is defined by the expression

$$
U_{j} \equiv \frac{1}{\lambda_{j}} \wp \nu_{j}=\frac{1}{\lambda_{j}} \sum_{k=1}^{s} A_{k} \nu_{j k} \in \mathbb{R}^{m \times n}, j \in \overline{1, r},
$$

where $\nu_{j k}$ are components of the vector $\nu_{j}^{T}=\left(\nu_{j 1}, \nu_{j 2}, \ldots, \nu_{j s}\right)$.

1.2. Singular decomposition of operators $\wp^{*}, \wp$. The operator singularity of $\wp^{*}, \wp$ uniquely determines decomposition of the singular operators $\wp^{*}, \wp$

$$
\wp y=\sum_{j=1}^{r} \lambda_{j} U_{j} \nu_{j}^{T} y, \quad y \in \mathbb{R}^{s}, \quad \wp^{*} Y=\sum_{j=1}^{r} \lambda_{j} \nu_{j}\left(U_{j}, Y\right)_{\mathrm{tr}}, \quad Y \in \mathbb{R}^{m \times n} .
$$

The pseudo inverse through the SVD representation of operators $\wp^{*}, \wp$ we determine by the relationships:

$$
\begin{gathered}
\left(\wp^{*}\right)^{+} y=\sum_{j=1}^{r} \lambda_{j}^{-1} U_{j} \nu_{j}^{T} y, y \in \mathbb{R}^{s}, \\
\wp^{+} Y=\sum_{j=1}^{r} \lambda_{j}^{-1} \nu_{j}\left(U_{j}, Y\right)_{\mathrm{tr}}, Y \in \mathbb{R}^{m \times n} .
\end{gathered}
$$

To represent the solution of the problem (1) formula (3) is used where the orthogonal projector $Z(\wp)$ on the operator core $\wp$, which is determined according to the expression

$$
Z(\wp)=E_{s}-P(\wp)
$$

and $P(\wp)=\wp^{+} \wp$.

Pseudo-inversion in matrix spaces has the properties defined by Penrose:

$$
\wp^{+} Y=\underset{\substack{A \\ \operatorname{Arg} \min \|\wp y-Y\|^{2}}}{\arg \min }\|y\|^{2}, \quad Y \in \mathbb{R}^{m \times n}, \quad Y \neq 0 .
$$

If the matrices $A_{k} \in \mathbb{R}^{m \times n}, k \in \overline{1, s}$ are perturbed (formula (2)), then it is necessary to get perturbed representations for the operator $\wp(\varepsilon): \mathbb{R}^{s} \rightarrow \mathbb{R}^{m \times n}$ and the adjoint operator $\wp^{*}(\varepsilon): \mathbb{R}^{m \times n} \rightarrow \mathbb{R}^{s}$ assuming that all parameters of the nonperturbed operator are known, and perturbation causes the change of singular numbers within the limits of the smaller minimum singular numbers of nonperturbed operator.

Since the operators and the pseudo-inversion of these operators are uniquely determined by the singularities (the set of eigenvalues and eigenvectors) of the operator, the solution of the problem is reduced to the determination of the set of singularities of the perturbed operator. 
Since the operators $\wp, \wp^{*}$ and the pseudo-inversion of these operators are uniquely determined by the singularities (the set of eigenvalues and eigenvectors) of the operator $\wp^{*} \wp$, then the solution of the problem is reduced to determination of the set of singularities of perturbed operator $\wp^{*}(\varepsilon) \wp(\varepsilon)\left(\nu_{j}(\varepsilon), \lambda_{j}^{2}(\varepsilon)\right), j \in \overline{1, r}$,

$$
r=\operatorname{rang} \wp^{*}(\varepsilon) \wp(\varepsilon)=\operatorname{rang} \wp^{*}(0) \wp(0) .
$$

We assume that perturbation does not change the order of the indices in the sequence of singular numbers of unperturbed $\wp^{*} \wp$.

2. Definition of singularity set $\left(\nu_{\mathbf{j}}(\varepsilon), \lambda_{\mathbf{j}}^{\mathbf{2}}(\varepsilon)\right), \mathbf{j} \in \overline{\mathbf{1}, \mathbf{r}}$ of perturbed operator $\wp^{*}(\varepsilon) \wp(\varepsilon)$ by small parameter method. The set of singularities $\left(\nu_{j}(\varepsilon), \lambda_{j}^{2}(\varepsilon)\right), j \in \overline{1, r}$ of perturbed operator $\wp^{*}(\varepsilon) \wp(\varepsilon)$ is determined by a singular decomposition for matrix

$$
F(\varepsilon)=\left(\begin{array}{ccc}
\left\langle A_{1}(\varepsilon) A_{1}(\varepsilon)\right\rangle & \cdots & \left\langle A_{1}(\varepsilon) A_{s}(\varepsilon)\right\rangle \\
\vdots & \ddots & \vdots \\
\left\langle A_{s}(\varepsilon) A_{1}(\varepsilon)\right\rangle & \cdots & \left\langle A_{s}(\varepsilon) A_{s}(\varepsilon)\right\rangle
\end{array}\right)=F(0)+\varepsilon F(1)+o(\varepsilon)
$$

this matrix is identified with this operator.

A singularity $\left(\nu_{j}(\varepsilon), \lambda_{j}^{2}(\varepsilon)\right), j \in \overline{1, r}$ of the perturbed operator $\wp^{*}(\varepsilon) \wp(\varepsilon)$ is an eigenvalue and an eigenvector solution of the symmetric matrix $F(\varepsilon)$ :

$$
\left(F(\varepsilon)-\lambda_{j}^{2}(\varepsilon) E_{K}\right) \nu_{j}(\varepsilon)=0, j \in \overline{1, r} .
$$

The eigenvalue and eigenvector problem in the general case does not have an analytic solution, but an approximate analytic solution can be obtained by a small parameter method $[8,9]$. This method can be used when problem operator is infinitesimally different from a certain operator, the problem solution with which is known.

According to the statement of the problem for $\varepsilon=0$ and for matrix $F(0)$ we know the set of singularities $\left(\nu_{j}(0), \lambda_{j}^{2}(0)\right), j \in \overline{1, r}$. For $\varepsilon \neq 0$ the set of singularities $\left(\nu_{j}(\varepsilon), \lambda_{j}^{2}(\varepsilon)\right), j \in$ $\overline{1, r}$ for the matrix $F(\varepsilon)=F(0)+\varepsilon F(1)$ according to the small parameter theory in the first approximation, we represent by the expressions:

$$
\left.\lambda_{j}^{2}(\varepsilon)\right)=\lambda_{j}^{2}(0)+\varepsilon \lambda_{j}^{2}(1)+o(\varepsilon), j \in \overline{1, r}, \quad \nu_{j}(\varepsilon)=\nu_{j}(0)+\varepsilon \nu_{j}(1)+o(\varepsilon), j \in \overline{1, r},
$$

It is necessary to determine the analytical corrections $\varepsilon \lambda_{j}^{2}(1)$ of singular numbers in the expressions, as well as analytical corrections $\varepsilon \nu_{i}(1)$ to singular vectors.

Solving the problem (7), we can have the case when singular number corresponds to a single singular vector. But we can have an alternative case when a singular number corresponds to a singular vector subspace. It is reasonable working with small parameter method to investigate the case of the close singular numbers.

2.1. Definition of singularity set $\left(\nu_{\mathbf{j}}(\varepsilon), \lambda_{\mathbf{j}}^{\mathbf{2}}(\varepsilon)\right), \mathbf{j} \in \overline{\mathbf{1}, \mathbf{r}}$ for simple eigenvalues. Substitution of expressions from (8) in (7), grouping members of development in the same degrees $\varepsilon$ allows us to obtain:

$$
\begin{gathered}
\varepsilon^{0}:\left(F(0)-\lambda_{j}^{2}(0) E_{K}\right) \nu_{j}(0)=0, \quad j \in \overline{1, r} \\
\varepsilon^{1}:\left(F(0)-\lambda_{j}^{2}(0) E_{K}\right) \nu_{j}(1)=-\left(F(1)-\lambda_{j}^{2}(1) E_{K}\right) \nu_{j}(0), j \in \overline{1, r} .
\end{gathered}
$$


According to the problem statement the system of vectors $\nu_{j}(0), j \in \overline{1, r}$ is orthonormal, forms a basis, the decomposition of which we can have eigenvectors of equality (8):

$$
\nu_{j}(1)=\sum_{i=1}^{r} c_{j i}^{1} \nu_{i}(0), j \in \overline{1, r}
$$

Taking into account decomposition (10) and relation (9) we have:

$$
\varepsilon^{1}: \sum_{i=1}^{r} c_{j i}^{1}\left(\lambda_{i}^{2}(0)-\lambda_{j}^{2}(0)\right) \nu_{i}(0)-\lambda_{j}^{2}(1) \nu_{j}(0)=-F(1) \nu_{j}(0), j \in \overline{1, r} .
$$

Vector expression (11) for the basic elements is converted into a scalar expression after the projection of the expression on a certain base vector $\nu_{q}(0), q \in \overline{1, r}$.

$$
\varepsilon^{1}: c_{j q}^{1}\left(\lambda_{q}^{2}(0)-\lambda_{j}^{2}(0)\right)-\lambda_{j}^{2}(1) \delta_{q j}=-F_{q j}(1), j \in \overline{1, r},
$$

where $F_{q j}(1)=\nu_{q}^{T}(0) F(1) \nu_{j}(0), q \in \overline{1, r}, j \in \overline{1, r}$.

From equation (12) we have the expressions

$$
\lambda_{j}^{2}(1)=F_{j j}(1), \quad c_{j q}^{1}=-F_{q j}(1) /\left(\lambda_{q}^{2}(0)-\lambda_{j}^{2}(0)\right), \quad q \in \overline{1, r}, \quad j \in \overline{1, r}, \quad q \neq j
$$

which allow us to write the development of the coefficients $\lambda_{j}^{2}(\varepsilon), \nu_{j}(\varepsilon), j \in \overline{1, r}(8)$ in the first approximation:

$$
\begin{gathered}
\lambda_{j}^{2}(\varepsilon)=\lambda_{j}^{2}(0)+\varepsilon F_{j j}(1)+o(\varepsilon), j \in \overline{1, r}, \quad \nu_{j}(\varepsilon)=\nu_{j}(0)+\varepsilon \nu_{j}(1)+o(\varepsilon) j \in \overline{1, r}, \\
\nu_{j}(1)=c_{j j}^{1} \nu_{j}(0)+\sum_{q=1, q \neq j}^{r} c_{j q}^{1} \nu_{q}(0), j \in \overline{1, r},
\end{gathered}
$$

where (it follows from the condition of normalization): $c_{j j}^{1}=0, j \in \overline{1, r}$.

2.2. Definition of a set of singularities $\left(\nu_{\mathbf{j}}(\varepsilon), \lambda_{\mathbf{j}}^{\mathbf{2}}(\varepsilon)\right), \mathbf{j} \in \overline{\mathbf{1}, \mathbf{r}}$ for multiple eigenvalues. We assume that to the eigenvalues $\lambda_{l}^{2}(0), l \in \overline{1, r}$ of matrix $F(0)$ correspond several orthonormal eigenvectors $\nu_{l 1}(0), \nu_{l 2}(0), \ldots, \nu_{l q}(0), l \in \overline{1, r}$, which can be considered as one of the bases of the subspace $P_{q}$ of the eigenvalue $\lambda_{l}^{2}(0), l \in \overline{1, r},(q$ degeneration rate). According to the perturbation method, we have

$$
\begin{gathered}
\varepsilon^{0}:\left(F(0)-\lambda_{l}^{2}(0) E_{K}\right) \nu_{l}(0)=0, \quad l \in \overline{1, r} \\
\varepsilon^{1}:\left(F(0)-\lambda_{l}^{2}(0) E_{K}\right) \nu_{l}(1)=-\left(F(1)-\lambda_{l}^{2}(1) E_{K}\right) \nu_{l}(0), l \in \overline{1, r}
\end{gathered}
$$

where from the equality (13) the vector $\nu_{l}(0)$, unlike the case of degeneration, is not uniquely determined, and it only indicates on its belonging to the subspace $P_{q}$.

We know that the solvability of equation (14) is determined by the condition of orthogonality of its right part to the subspace $P_{q}$, that is, the equality

$$
H\left(F(1)-\lambda_{l}^{2}(1) E_{K}\right) \nu_{l}(0)=0, l \in \overline{1, r}
$$

holds, where $H=Z\left(F(0)-\lambda_{l}^{2}(0) E_{K}\right) \equiv E_{K}-\left(F(0)-\lambda_{l}^{2}(0) E_{K}\right)^{T}\left(F(0)-\lambda_{l}^{2}(0) E_{K}\right), l \in \overline{1, r}$. 
Taking into account the operator action $H: H \nu_{l}(0)=\nu_{l}(0), l \in \overline{1, r}$, we rewrite equality (15) as an equation for functions $\nu_{l}(0), l \in \overline{1, r}$ :

$$
H\left(F(1) H \nu_{l}(0)=\lambda_{l}^{2}(1) \nu_{l}(0), l \in \overline{1, r} .\right.
$$

In the case of multiple elements $\lambda_{l i}^{2}(1), l \in \overline{1, r}, i \in \overline{1, q}$ of the perturbed operator we have the representation for the first approximation:

$$
\lambda_{l j}^{2}(\varepsilon)=\lambda_{l}^{2}(0)+\varepsilon \lambda_{l j}^{2}(1)+o(\varepsilon), \quad l \in \overline{1, r}, j \in \overline{1, q},
$$

and orthonormal solutions of the system (16) give us the determination of the equations (14) of the vectors $\nu_{l}^{i}(1) l \in \overline{1, q}, i \in \overline{1, q}$.

3. The first approximation of perturbed singular decompositions of operators $\wp^{*}, \wp$. For the operator $\wp^{*}(\varepsilon): \mathbb{R}^{m \times n} \rightarrow \mathbb{R}^{s}$ the elements of the complete set of singularities are $\left(U_{j}(\varepsilon), \lambda_{j}^{2}(\varepsilon)\right), j \in \overline{1, r}$, where the matrices $U_{j}(\varepsilon) \in \mathbb{R}^{m \times n}$, are determined according to the formulas:

$$
\begin{gathered}
U_{j}(\varepsilon)=\frac{1}{\lambda_{j}(\varepsilon)} \sum_{k=1}^{s} A_{k}(\varepsilon) \nu_{j k}(\varepsilon)=U_{j}(0)+\varepsilon U_{j}(1)+o(\varepsilon), \\
\lambda_{j}^{-1}(\varepsilon)=\lambda_{j}^{-1}(0)\left(1-\varepsilon \lambda_{j}^{2}(1) \lambda_{j}^{-2}(0) / 2\right)+o(\varepsilon), \quad A_{k}(\varepsilon)=A_{k}+\varepsilon A_{k}(1)+o(\varepsilon), \\
\nu_{j k}(\varepsilon)=\nu_{j k}(0)+\varepsilon \nu_{j k}(1)+o(\varepsilon), \quad \nu_{j k}(1)=\sum_{q=1, q \neq j}^{r} c_{q j}(1) \nu_{j k}(0), \\
U_{j}(0)=\frac{1}{\lambda_{j}(0)} \sum_{k=1}^{s} A_{k} \nu_{j k}(0), \\
U_{j}(1)=\frac{1}{\lambda_{j}(0)} \sum_{k=1}^{s}\left(A_{k} \nu_{j k}(1)+A_{k}(1) \nu_{j k}(0)\right)-\lambda_{j}(0) \lambda_{j}^{2}(1) U_{j}(0) / 2, \quad j \in \overline{1, r}, k \in \overline{1, s}
\end{gathered}
$$

Formulas (17)-(20) allow to determine the singular operator decomposition $\wp^{*}(\varepsilon), \wp(\varepsilon)$ in the first approximation:

$$
\begin{gathered}
\wp(\varepsilon) y=\wp y+\varepsilon \wp(1) y+o(\varepsilon), y \in \mathbb{R}^{s}, \quad \wp y=\sum_{j=1}^{r} \lambda_{j}(0) U_{j}(0) \nu_{j}^{T}(0) y, y \in \mathbb{R}^{s}, \\
\wp(1) y=\sum_{j=1}^{r}\left(\lambda_{j}(1) U_{j}(0) \nu_{j}^{T}(0)+\lambda_{j}(0) U_{j}(1) \nu_{j}^{T}(0)+\lambda_{j}(0) U_{j}(0) \nu_{j}^{T}(1)\right) y, \\
\wp^{*}(\varepsilon) Y=\wp^{*} Y+\varepsilon \wp^{*}(1) Y+o(\varepsilon), \quad Y \in \mathbb{R}^{m \times n}, \wp^{*}(\varepsilon) Y=\wp^{*} Y+\varepsilon \wp^{*}(1) Y+o(\varepsilon), Y \in \mathbb{R}^{m \times n}, \\
\wp^{*} Y=\sum_{j=1}^{r} \lambda_{j}(0) \nu_{j}(0)\left(U_{j}(0), Y\right)_{\operatorname{tr}}, Y \in \mathbb{R}^{m \times n}, \\
\wp^{*}(1) Y=\sum_{j=1}^{r}\left(\lambda_{j}(1) \nu_{j}(0)\left(U_{j}(0), Y\right)_{\operatorname{tr}}+\lambda_{j}(0) \nu_{j}(1)\left(U_{j}(0), Y\right)_{\operatorname{tr}}+\lambda_{j}(0) \nu_{j}(0)\left(U_{j}(1), Y\right)_{\operatorname{tr}}\right) .
\end{gathered}
$$

4. The first approximation through SVD representation of perturbed pseudo inverse operators $\wp^{+}(\varepsilon),\left(\wp^{*}(\varepsilon)\right)^{+}$. The pseudo inverse through the SVD representation of perturbed operators $\wp^{*}(\varepsilon), \wp(\varepsilon)$ is determined by the relationships:

$$
\left(\wp^{*}(\varepsilon)\right)^{+} y=\left(\wp^{*}\right)^{+} y+\varepsilon\left(\wp^{*}(1)\right)^{+} y+o(\varepsilon), y \in \mathbb{R}^{s}
$$




$$
\begin{gathered}
\left(\wp^{*}\right)^{+} y=\sum_{j=1}^{r} \lambda_{j}^{-1}(0) U_{j}(0) \nu_{j}^{T}(0) y, \quad y \in \mathbb{R}^{s}, \\
\left(\wp^{*}(1)\right)^{+} y=\sum_{j=1}^{r}\left(\lambda_{j}^{-1}(1) U_{j}(0) \nu_{j}^{T}(0)+\gamma_{j}(1)+\lambda_{j}^{-1}(0) U_{j}(0) \nu_{j}^{T}(1)\right) y, \\
\gamma_{j}(1)=\lambda_{j}^{-1}(0) U_{j}(1) \nu_{j}^{T}(0), j \in \overline{1, r}, y \in \mathbb{R}^{s}, \\
\wp^{+}(\varepsilon) Y=\wp^{+} Y+\varepsilon \wp^{+}(1) Y+o(\varepsilon), \quad Y \in \mathbb{R}^{m \times n}, \\
\wp^{+} Y=\sum_{j=1}^{r} \lambda_{j}^{-1}(0) \nu_{j}(0)\left(U_{j}(0), Y\right)_{\operatorname{tr}}, \quad Y \in \mathbb{R}^{m \times n}, \\
\wp^{+}(1) Y=\sum_{j=1}^{r}\left(\mu_{j}(1)+\lambda_{j}^{-1}(0) \nu_{j}(1)\left(U_{j}(0), Y\right)_{\operatorname{tr}}+\lambda_{j}^{-1}(0) \nu_{j}(0)\left(U_{j}(1), Y\right)_{\operatorname{tr}}\right), \\
\mu_{j}(1)=\lambda_{j}^{-1}(1) \nu_{j}(0)\left(U_{j}(0), Y\right)_{\operatorname{tr}}, \quad j \in \overline{1, r}, \quad Y \in \mathbb{R}^{m \times n} .
\end{gathered}
$$

Representation in the first approximation for the main orthogonal projectors (relations (6), (7)) are obtained using formulas (21), (22).

5. Example. The problem of finding the solution in the first approximation of the matrix equation is considered

$$
y_{1}(\varepsilon)\left(A_{1}+\varepsilon A_{1}(1)\right)+y_{2}(\varepsilon)\left(A_{2}+\varepsilon A_{2}(1)\right)=Y \Leftrightarrow \quad \wp(\varepsilon) y(\varepsilon)=Y \Leftrightarrow y(\varepsilon)=\wp^{+}(\varepsilon) Y,
$$

where $Y \in \mathbb{R}^{m \times n}, A_{k}(\varepsilon)=\left(A_{k}+\varepsilon A_{k}(1)\right) \in \mathbb{R}^{m \times n}, k=\overline{1,2}$ given matrices, $y_{1}(\varepsilon), y_{2}(\varepsilon)$ unknown real numbers,

$$
\begin{gathered}
y(\varepsilon) \equiv\left(y_{1}(\varepsilon), y_{2}(\varepsilon)\right)^{T}=\left(y_{1}+\varepsilon y_{1}(1), y_{2}+\varepsilon y_{2}(1)\right)^{T} \in \mathbb{R}^{2}, \\
\wp(\varepsilon) y(\varepsilon)=\sum_{k=1}^{2} y_{k}(\varepsilon)\left(A_{k}+\varepsilon A_{k}(1)\right), \wp^{+}(\varepsilon) Y=\wp^{+} Y+\varepsilon \wp^{+}(1) Y .
\end{gathered}
$$

\section{The solution:}

$$
y(\varepsilon)=\wp^{+} Y+\varepsilon \wp^{+}(1) Y+\left(E_{2}-\left(\wp^{+} Y+\varepsilon \wp^{+}(1) Y\right)(\wp y+\varepsilon \wp(1) y)\right) w+o(\varepsilon), w \in \mathbb{R}^{2} .
$$

Formulas to determine $\wp^{+} Y, \wp^{+}(1), \wp y, \wp(1) y$ are given below.

Proof. Using the technique of the calculation proposed above

$$
\begin{aligned}
& F(\varepsilon)=F(0)+\varepsilon F(1)+o(\varepsilon), \\
& F(0)=\left(\begin{array}{ll}
\left\langle A_{1}(0) A_{1}(0)\right\rangle & \left\langle A_{1}(0) A_{2}(0)\right\rangle \\
\left\langle A_{2}(0) A_{1}(0)\right\rangle & \left\langle A_{2}(0) A_{2}(0)\right\rangle
\end{array}\right) \equiv\left(\begin{array}{ll}
f_{11} & f_{12} \\
f_{12} & f_{22}
\end{array}\right), \\
& F(1)=\left(\begin{array}{cc}
2\left\langle A_{1}(0) A_{1}(1)\right\rangle & \left\langle A_{1}(0) A_{2}(1)\right\rangle+\left\langle A_{1}(1) A_{2}(0)\right\rangle \\
\left\langle A_{2}(0) A_{1}(1)\right\rangle+\left\langle A_{2}(1) A_{1}(0)\right\rangle & 2\left\langle A_{2}(1) A_{2}(0)\right\rangle
\end{array}\right) \equiv \\
& \equiv\left(\begin{array}{ll}
f_{11}(1) & f_{12}(1) \\
f_{12}(1) & f_{22}(1)
\end{array}\right)
\end{aligned}
$$

To simplify the calculations we assume that $f_{11} \neq f_{22}, f_{12}=0$.

$$
\begin{aligned}
& \lambda_{1}^{2}(0)=f_{11}, \quad \lambda_{1}^{2}(1)=f_{22}(1), \Rightarrow \lambda_{1}^{-1}(0)=f_{11}^{-1 / 2}, \quad \lambda_{1}^{-1}(1)=f_{22}(1) f_{11}^{-3 / 2} / 2, \\
& \lambda_{2}^{2}(0)=f_{22}, \quad \lambda_{2}^{2}(1)=f_{11}(1), \Rightarrow \lambda_{2}^{-1}(0)=f_{22}^{-1 / 2}, \quad \lambda_{2}^{-1}(1)=f_{11}(1) f_{22}^{-3 / 2} / 2,
\end{aligned}
$$




$$
\begin{gathered}
\nu_{1}(0)=\left(\begin{array}{cc}
0, & 1
\end{array}\right)^{T}, \quad \nu_{2}(0)=\left(\begin{array}{cc}
1, & 0
\end{array}\right)^{T} \\
\nu_{1}(1)=\left(f_{11}(1) /\left(f_{11}-f_{22}\right)\right)\left(\begin{array}{cc}
1, & 0
\end{array}\right)^{T}, \quad \nu_{2}(1)=\left(f_{22}(1) /\left(f_{22}-f_{11}\right)\right)\left(\begin{array}{ll}
0, & 1
\end{array}\right)^{T}, \\
U_{1}(0)=\frac{1}{\lambda_{1}(0)} A_{2}, \quad U_{2}(0)=\frac{1}{\lambda_{2}(0)} A_{1}, \\
U_{1}(1)=\frac{1}{\lambda_{1}(0)}\left(\left(f_{11}(1) /\left(f_{11}-f_{22}\right)\right) A_{1}+A_{2}(1)\right)-\lambda_{1}^{2}(1) A_{2} / 2, \\
U_{2}(1)=\frac{1}{\lambda_{2}(0)}\left(A_{1}(1)+\left(f_{22}(1) /\left(f_{22}-f_{11}\right)\right) A_{2}\right)-\lambda_{2}^{2}(1) A_{1} / 2 .
\end{gathered}
$$

We verify orthonormality of own matrices of adjoint operator:

$$
\begin{gathered}
\left\langle U_{j}(0)+\varepsilon U_{j}(1)+o(\varepsilon), U_{j}(0)+\varepsilon U_{j}(1)+o(\varepsilon)\right\rangle=1+o(\varepsilon), \quad j \in \overline{1,2} \\
\left\langle U_{1}(0)+\varepsilon U_{1}(1)+o(\varepsilon), U_{2}(0)+\varepsilon U_{2}(1)+o(\varepsilon)\right\rangle=>(\varepsilon) .
\end{gathered}
$$

SVD representation of the perturbed operator $\wp(\varepsilon)$

$$
\begin{gathered}
\wp y=\sum_{j=1}^{2} \lambda_{j}(0) U_{j}(0) \nu_{j}^{T}(0) y, \quad y \in \mathbb{R}^{2}, \\
\wp(1) y=\sum_{j=1}^{r}\left(\lambda_{j}(1) U_{j}(0) \nu_{j}^{T}(0)+\lambda_{j}(0) U_{j}(1) \nu_{j}^{T}(0)+\lambda_{j}(0) U_{j}(0) \nu_{j}^{T}(1)\right) y, \quad y \in \mathbb{R}^{s} .
\end{gathered}
$$

Pseudo inverse through SVD representation of the perturbed operator $\wp(\varepsilon)$ :

$$
\begin{gathered}
\wp^{+} Y=\sum_{j=1}^{2} \lambda_{j}^{-1}(0) \nu_{j}(0)\left(U_{j}(0), Y\right)_{\mathrm{tr}}, Y \in \mathbb{R}^{m \times n} \\
\wp^{+}(1) Y=\sum_{j=1}^{2}\left(\mu_{j}(1)+\lambda_{j}^{-1}(0) \nu_{j}(1)\left(U_{j}(0), Y\right)_{\mathrm{tr}}+\lambda_{j}^{-1}(0) \nu_{j}(0)\left(U_{j}(1), Y\right)_{\mathrm{tr}}\right) \\
\mu_{j}(1)=\lambda_{j}^{-1}(1) \nu_{j}(0)\left(U_{j}(0), Y\right)_{\mathrm{tr}}, j \in \overline{1,2} .
\end{gathered}
$$

6. Conclusion. We investigated a linear operator equation, when the operator is a linear combination of matrices. Introducing the adjoint operator, we performed the solution of the problem in a matrix space using a pseudo inverse operation. We obtained the solution of the problem using the pseudo inverse operation with the presence of small perturbations of matrices from the set of matrices of the operator, using small parameter method; in the first approximation, it contains all the necessary analytical expressions.

\section{REFERENCES}

1. Albert A. Regression, pseudo inverse, recurrent evaluation. - M .: Nauka, 1977. - 305 p. (in Russian) 
2. Cline R.E. Representation for the generalized inverse of partitioned matrix// SIAM J. Appl. Math. - 1964. - V.32. - P. 588-600.

3. Kirichenko N.F. An analytical representation of perturbations of pseudo inverse matrices// Cybernetics and system analysis. - 1997. - №2. - P. 98-107. (in Russian)

4. Kirichenko N.F., Donchenko V.S. Pseudo Reversal in the problems of clusterization// Cybernetics and System Analysis. - 2007. - №4. - P. 73-92. (in Russian)

5. Kirichenko N.F., Kudin G.I. Analysis and synthesis of systems of classification of signals by means of perturbations of pseudo inverse and projection operations// Cybernetics and system analysis. - 2009. №3. - P. 47-57. (Russian)

6. Kirichenko N.F., Kudin G.I. Variations of pseudo inverse and projection matrices with perturbation of elements of the original matrix// Problems of control and informatics. - 2009. - №3. - P. 7-20. (in Russian)

7. Donchenko V.S., Zinko T., Skotarenko F. 'Feature vectors' in grouping information problem in applied mathematics: vectors and matrices// Problems of Computer Intellectualization, Kyiv: ITHEA, 2012. P. 111-124.

8. Nayfe A.H. Introduction to perturbation methods. - M.: Mir, 1984. - 536 p. (in Russian)

9. Blokhintsev D.I. Fundamentals of Quantum Mechanics. - M .: Nauka, 1976. - 864 p. (in Russian)

Taras Shevchenko National University of Kyiv

Kyiv, Ukraine

a.nakonechniy@gmail.com

gkudin@ukr.net

taras.zinko@gmail.com 